\title{
Fuzzy Energy-Based Active Contours Exploiting Local Information
}

\author{
Stelios Krinidis and Michail Krinidis \\ Information Management Department \\ Technological Institute of Kavala, \\ Ag. Loukas, 65404 Kavala, Greece \\ stelios.krinidis@mycosmos.gr, mkrinidi@gmail.com
}

\begin{abstract}
This paper presents a novel fast and robust model for active contours to detect objects in an image, based on techniques of curve evolution. The proposed model can detect objects whose boundaries are not necessarily defined by gradient, based on the minimization of a fuzzy energy. This fuzzy energy is used as the model motivation power evolving the active contour, which will stop on the desired object boundary. The fuzziness of the energy provides a balanced technique with a strong ability to reject "weak", as well as, "strong" local minima. Also, this approach differs from previous methods, since it does not solve the EulerLagrange equations of the underlying problem, but, instead, calculates the fuzzy energy alterations directly. So, it converges to the desired object boundary very fast. The theoretical properties and various experiments presented demonstrate that the proposed fuzzy energy-based active contour is better and more robust than classical snake methods based on the gradient or other kind of energies.
\end{abstract}

Keywords: Active contour, deformable curve, curve evolution, fuzzy logic, energy-based.

\section{Introduction}

Image segmentation is one of the first and most important tasks in image analysis and computer vision [1]. The design of robust and efficient segmentation algorithms is still a very challenging research topic, due to the variety and complexity of images.

Since the introduction of snakes 2], active contours have been applied to a variety of problems in image processing and computer vision such as segmentation and feature extraction, visual tracking, etc. The basic idea in active contour models or snakes is to evolve a curve, in order to detect objects in the image under consideration. For instance, starting with a curve around the object to be detected, the curve moves toward its interior normal and has to stop on the boundary of the object.

Geometric active contour models [3], where introduced shortly afterwards, based on curve evolution theory, which could also handle topology changes very

L. Iliadis et al. (Eds.): AIAI 2012, IFIP AICT 381, pp. 175-184, 2012.

(c) IFIP International Federation for Information Processing 2012 
naturally, when implemented using level set methods proposed by Osher and Sethian [4].

The geometric active contour model, which is most related to the original snake model, is probably the geodesic active contour model [5, 6], which has been based on the curvature of the image and on an inflationary force [7].

Cohen et al. [8] have proposed the minimal path technique, a semi-automatic method, which captures the global minimum of a contour energy between two fixed user-defined end points, exploiting an oriented graph characterized by its cost function. The object boundary detection becomes the optimal path search problem between two user-defined points in the graph. This approach leads the snake-like energy to a global minimum, avoiding any local minima.

Other implementations have also been proposed for capturing more global minimizers by restricting the search space. Dual snakes, proposed by Gunn and Nixon [9, 10], is such a method. This method uses two interlinked snakes instead of one. Similar methods were also proposed in [11 13]. These methods are restricted to detection of objects with simple topologies. Also, active contours have been combined with the optimization tool of graph-cuts [14], such as morphological dilation in order to restrict the search space for graph-cuts segmentation. However, this method cannot handle images with multiple objects simultaneously.

All these classical snakes and active contour models are known as "edgebased" models, since they rely on edge-functionals to stop the curve evolution. Also, these models can detect only objects with edges defined by gradient. The performance of the purely edge-based models is often inadequate. There has been much research into the design of complex region-based energy functionals [1519] utilizing image information not only near the evolving contour, but image statistics inside and outside the contour as well in order to achieve better performance. Unfortunately, most of these region-based energy functionals assume highly constrained models for pixel intensities within each region.

This paper deals with the above mentioned problems. It presents a novel, fast and robust fuzzy energy-based active contour for image segmentation, which can handle objects whose boundaries are not necessarily defined by gradient. Generally, fuzzy methods provide more accurate and robust data clustering, thus, we combine it with active contour methodology, introducing here a model as a fuzzy energy-based minimization. The fuzziness of the energy provides a balanced technique with a strong ability to reject "weak", as well as, "strong" local minima. Furthermore, we formulate the model in terms of pseudo-level set functions, but instead of computing the associated Euler-Lagrange equations, we apply a direct method to solve the corresponding equations without numerical stability constraints.

The remainder of the paper is organized as follows. The description of the model and its fuzzy motivation energy is presented in Section 2, Experimental results are presented in Section 3 and conclusions are drawn in Section 4 . 


\section{$2 \quad$ Fuzzy Energy-Based Model}

Let us define the evolving curve $C$ in the image domain $\Omega$. The proposed approach is based on the minimization of a fuzzy energy-based segmentation. Firstly, let us assume that the image $I$ is formed by two regions of approximately piecewise-constant intensities and the object to be detected is represented by a region and its boundary by $C_{0}$. Now, let us consider the following functionals:

$$
\begin{aligned}
& F_{1}(C)+F_{2}(C) \\
& =\int_{\Omega}[u(x, y)]^{m}\left[\left|I(x, y)-c_{1}\right|^{2}+\int_{\Omega} w_{x y, x^{\prime} y^{\prime}} R_{1}\left(x^{\prime}, y^{\prime}\right) d x^{\prime} d y^{\prime}\right] d x d y \\
& \quad+\int_{\Omega}[1-u(x, y)]^{m}\left[\left|I(x, y)-c_{2}\right|^{2}+\int_{\Omega} w_{x y, x^{\prime} y^{\prime}} R_{2}\left(x^{\prime}, y^{\prime}\right) d x^{\prime} d y^{\prime}\right] d x d y
\end{aligned}
$$

where $c_{1}$ and $c_{2}$ are constants depending on $C$, expressing the average prototypes of the image regions inside and outside respectively of $C$. The membership function $u(x, y) \in[0,1]$ is the degree of membership of $I(x, y)$ to the inside of $C$, and $m$ is a weighting exponent on each fuzzy membership. The term $w_{x y, x^{\prime} y^{\prime}}$ incorporates the spatial dependence between the image points, that is, a term that quantifies the degree of spatial contiguity of the points $I(x, y)$ and $I\left(x^{\prime}, y^{\prime}\right)$. The spatial structure of a given data set is defined by using a matrix $W$ :

$$
w_{x y, x^{\prime} y^{\prime}}= \begin{cases} & \text { if } I(x, y) \text { and } I\left(x^{\prime}, y^{\prime}\right) \text { are } \\ \frac{1}{d_{x y, x^{\prime} y^{\prime}}+1}, & \text { neighbors and } \\ & {[x, y]^{T} \neq\left[x^{\prime}, y^{\prime}\right]^{T}} \\ 0, & \text { otherwise }\end{cases}
$$

where $d_{x y, x^{\prime} y^{\prime}}$ is the distance between points $I(x, y)$ and $I\left(x^{\prime}, y^{\prime}\right)$. Also, terms $R_{1}\left(x^{\prime}, y^{\prime}\right)$ and $R_{2}\left(x^{\prime}, y^{\prime}\right)$ are used for regularizing the data set points among the clusters, and given by:

$$
\begin{aligned}
& R_{1}\left(x^{\prime}, y^{\prime}\right)=\left[1-u\left(x^{\prime}, y^{\prime}\right)\right]^{m}\left|I\left(x^{\prime}, y^{\prime}\right)-c_{1}\right|^{2} \\
& R_{2}\left(x^{\prime}, y^{\prime}\right)=\left[u\left(x^{\prime}, y^{\prime}\right)\right]^{m}\left|I\left(x^{\prime}, y^{\prime}\right)-c_{2}\right|^{2} .
\end{aligned}
$$

In this simple case, it is obvious that the boundary of the object $C_{0}$, is the minimizer of the "fitting" term:

$$
\inf _{C}\left\{F_{1}(C)+F_{2}(C)\right\} \approx 0 \approx F_{1}\left(C_{0}\right)+F_{2}\left(C_{0}\right) .
$$

The proposed active contour is based on the minimization of the above fitting term, taking into account the length of the model $C$ as a regularization term. Therefore, the energy functional $F\left(C, c_{1}, c_{2}, u\right)$ is introduced as:

$$
\begin{aligned}
& F\left(C, c_{1}, c_{2}, u\right) \\
& =\mu \cdot \operatorname{Length}(C)
\end{aligned}
$$




$$
\begin{aligned}
& +\lambda_{1} \int_{\Omega}[u(x, y)]^{m}\left[\left|I(x, y)-c_{1}\right|^{2}+\int_{\Omega} w_{x y, x^{\prime} y^{\prime}} R_{1}\left(x^{\prime}, y^{\prime}\right) d x^{\prime} d y^{\prime}\right] d x d y \\
& +\lambda_{2} \int_{\Omega}[1-u(x, y)]^{m}\left[\left|I(x, y)-c_{2}\right|^{2}+\int_{\Omega} w_{x y, x^{\prime} y^{\prime}} R_{2}\left(x^{\prime}, y^{\prime}\right) d x^{\prime} d y^{\prime}\right] d x d y
\end{aligned}
$$

where $\mu \geq 0, \lambda_{1}, \lambda_{2}>0$ are fixed parameters. The curve $C_{0}$ that minimizes $F$ :

$$
F\left(C_{0}, c_{1}, c_{2}, u\right)=\inf _{C} F\left(C, c_{1}, c_{2}, u\right),
$$

is the solution to the segmentation problem (object boundary). The first term, in the definition of $F$ (4), accounts for smoothing of the curve $C$.

\subsection{Pseudo Level-Set Formulation}

Let us define a pseudo level set formulation, similar to the level set method[4], based on the membership values $u$, where $C \subset \Omega$ is represented by the pseudo zero level set of Lipschitz similar function $u: \Omega \rightarrow \mathbb{R}$, such that:

$$
u(x, y)= \begin{cases}C & =\{(x, y) \in \Omega: u(x, y)=0.5\} \\ \text { inside }(C)=\{(x, y) \in \Omega: u(x, y)>0.5\} \\ \text { outside }(C)=\{(x, y) \in \Omega: u(x, y)<0.5\}\end{cases}
$$

For more details, we refer the reader to Osher et al. [4].

We could express the regularization term of the energy $F$ as Length $(C)=$ $\int_{\Omega}|\nabla H(u(x, y)-0.5)| d x d y$, exploiting the Heaviside function $H(s)$ [4]. Thus, the energy $F\left(C, c_{1}, c_{2}, u\right)$ (4) can be rewritten as:

$$
\begin{aligned}
& F\left(C, c_{1}, c_{2}, u\right) \\
& =\mu \int_{\Omega}|\nabla H(u(x, y)-0.5)| d x d y \\
& \quad+\lambda_{1} \int_{\Omega}[u(x, y)]^{m}\left[\left|I(x, y)-c_{1}\right|^{2}+\int_{\Omega} w_{x y, x^{\prime} y^{\prime}} R_{1}\left(x^{\prime}, y^{\prime}\right) d x^{\prime} d y^{\prime}\right] d x d y \\
& \quad+\lambda_{2} \int_{\Omega}[1-u(x, y)]^{m}\left[\left|I(x, y)-c_{2}\right|^{2}+\int_{\Omega} w_{x y, x^{\prime} y^{\prime}} R_{2}\left(x^{\prime}, y^{\prime}\right) d x^{\prime} d y^{\prime}\right] d x d y .
\end{aligned}
$$

Keeping $u$ fixed and minimizing the energy $F\left(C, c_{1}, c_{2}, u\right)$ (7) with respect to $c_{1}$ and and $c_{2}$, it is easy to express these constants functions of $u$ by:

$$
\begin{aligned}
c_{1}= & \frac{\int_{\Omega}[u(x, y)]^{m} I(x, y) d x d y}{\int_{\Omega}[u(x, y)]^{m} d x d y}, \\
c_{2}= & \frac{\int_{\Omega}[1-u(x, y)]^{m} I(x, y) d x d y}{\int_{\Omega}[1-u(x, y)]^{m} d x d y} .
\end{aligned}
$$


Furthermore, keeping $c_{1}$ and $c_{2}$ fixed and minimizing the energy $F\left(C, c_{1}, c_{2}, u\right)$ (17) with respect to $u$, it is easy to express variable $u$ in the following way:

$$
u(x, y)=\frac{1}{1+\left(\frac{\lambda_{1}\left[\left[\left|I(x, y)-c_{1}\right|^{2}+\int_{\Omega} w_{x y, x^{\prime} y^{\prime}} R_{1}\left(x^{\prime}, y^{\prime}\right) d x^{\prime} d y^{\prime}\right]\right]}{\left.\lambda_{2}\left[|| I(x, y)-\left.c_{2}\right|^{2}+\int_{\Omega} w_{x y, x^{\prime} y^{\prime}} R_{2}\left(x^{\prime}, y^{\prime}\right) d x^{\prime} d y^{\prime}\right]\right]}\right)^{\frac{1}{m-1}}} .
$$

For simplicity, without losing the generality, the above minimization (9) has been considered without the length term $(\mu=0)$.

\subsection{Numerical Approximation}

In equation (7), the two fitting terms are easy to be computed directly. We can also, approximate the length term $\int_{\Omega}|\nabla H(u(x, y)-0.5)| d x d y$ by:

$$
\sum_{i, j} \sqrt{\left(H\left(u_{i+1, j}-0.5\right)-H\left(u_{i, j}-0.5\right)\right)^{2}+\left(H\left(u_{i, j+1}-0.5\right)-H\left(u_{i, j}-0.5\right)\right)^{2}},
$$

where $u_{i, j}$ is the value of $u$ at the $(i, j)$ pixel. The summand can only take the values 0,1 , or $\sqrt{2}$, depending on whether the 3 distinct pair of points from the set $\left\{u_{i, j}, u_{i+1, j}, u_{i, j+1}\right\}$ belong to the same or different regions. Thus, the length term can be easily computed knowing only the $H(u-0.5)$, and there is no need to know $u$.

The usual approach to solve a minimization problem as in (17), is to derive its Euler-Lagrange equation and then to use explicit time marching or implicit iteration. In the proposed method, the time step is not restricted as in the explicit time marching. The algorithm for the fuzzy energy model is:

1. Give an initial partition of the image, set $u>0.5$ for one part and $u<0.5$ for the other.

2. Compute $c_{1}$ and $c_{2}$ using (8).

3. Assume that the value of the current pixel is $I_{o}$ and $u_{o}$ its corresponding degree of membership. Calculate the new degree of membership $u_{n}$ using (9) for the pixel $I_{o}$ under consideration and then compute the difference between the new and the old energy $\Delta F$ defined as:

$$
\begin{aligned}
& \Delta F= \\
& G\left[\lambda_{1}\left(s_{1}\left(I_{o}-c_{1}\right)+c_{1}\right)^{2}+\lambda_{2}\left(s_{2}\left(I_{o}-c_{2}\right)+c_{2}\right)^{2}-c_{1}^{2}-c_{2}^{2}\right] \\
& -2 \lambda_{1} s_{1}\left(I_{o}-c_{1}\right) \sum_{\Omega}\left[u(i, j)^{m}\left(\sum_{\Omega} w_{i j, i^{\prime} j^{\prime}}\left[1-u\left(i^{\prime}, j^{\prime}\right)\right]^{m} I\left(i^{\prime}, j^{\prime}\right)\right)\right] \\
& -2 \lambda_{2} s_{2}\left(I_{o}-c_{2}\right) \sum_{\Omega}\left[[1-u(i, j)]^{m}\left(\sum_{\Omega} w_{i j, i^{\prime} j^{\prime}} u\left(i^{\prime}, j^{\prime}\right)^{m} I\left(i^{\prime}, j^{\prime}\right)\right)\right] \\
& +\left(u_{n}^{m}-u_{o}^{m}\right) \sum_{\Omega} w_{i_{o} j_{o}, i^{\prime} j^{\prime}}\left[1-u\left(i^{\prime}, j^{\prime}\right)\right]^{m}\left[\lambda_{1}\left(I\left(i^{\prime}, j^{\prime}\right)-c_{1}-s_{1}\left(I_{o}-c_{1}\right)\right)^{2}\right] \\
& +\left(u_{n}^{m}-u_{o}^{m}\right) \sum_{\Omega} w_{i_{o} j_{o}, i^{\prime} j^{\prime}}\left[1-u\left(i^{\prime}, j^{\prime}\right)\right]^{m}\left[\lambda_{2}\left(I_{o}-c_{2}\right)^{2}\left(1-s_{2}\right)^{2}\right]
\end{aligned}
$$




$$
\begin{aligned}
& +\left[\left(1-u_{n}\right)^{m}-\left(1-u_{o}\right)^{m}\right] \sum_{\Omega} w_{i_{o} j_{o}, i^{\prime} j^{\prime}} u\left(i^{\prime}, j^{\prime}\right)^{m}\left[\lambda_{2}\left(I\left(i^{\prime}, j^{\prime}\right)-c_{2}-s_{2}\left(I_{o}-c_{2}\right)\right)^{2}\right] \\
& +\left[\left(1-u_{n}\right)^{m}-\left(1-u_{o}\right)^{m}\right] \sum_{\Omega} w_{i_{o} j_{o}, i^{\prime} j^{\prime}} u\left(i^{\prime}, j^{\prime}\right)^{m}\left[\lambda_{1}\left(I_{o}-c_{1}\right)^{2}\left(1-s_{1}\right)^{2}\right] \\
& +\left(u_{n}^{m}-u_{o}^{m}\right)\left(1-s_{1}\right)\left(I_{o}-c_{1}\right)^{2}+\left[\left(1-u_{n}\right)^{m}-\left(1-u_{o}\right)^{m}\right]\left(1-s_{2}\right)\left(I_{o}-c_{2}\right)^{2},(11)
\end{aligned}
$$

where terms $G, s_{1}$ and $s_{2}$ defined as:

$$
\begin{aligned}
G & =\sum_{i, j} u(i, j)^{m}\left[\sum_{\Omega} w_{i j, i^{\prime} j^{\prime}}\left[1-u\left(i^{\prime}, j^{\prime}\right)\right]^{m}\right] \\
s_{1} & =\frac{u_{n}^{m}-u_{o}^{m}}{\sum_{i, j}[u(i, j)]^{m}+u_{m}^{m}-u_{o}^{m}} \\
s_{2} & =\frac{\left(1-u_{n}\right)^{m}-\left(1-u_{o}\right)^{m}}{\sum_{i, j}[1-u(i, j)]^{m}+\left(1-u_{n}\right)^{m}-\left(1-u_{o}\right)^{m}}
\end{aligned} .
$$

If $\Delta F<0$, then change $u_{o}$ with $u_{n}$ value, else keep the old $\left(u_{o}\right)$ one. If we consider the length term $(\mu \neq 0)$, it is easy to be computed and incorporated into the above energy difference, since only four neighbor pixels will be affected, when we change the value of a pixel.

4. Calculate the new centers using equations:

$$
\begin{aligned}
& \tilde{c_{1}}=c_{1}+s_{1}\left(I_{o}-c_{1}\right) \\
& \tilde{c_{2}}=c_{2}+s_{2}\left(I_{o}-c_{2}\right) .
\end{aligned}
$$

5. Repeat the step 3 using Jacobi iterations computing the total energy $F$ of the image.

6. Repeat the steps 2 to 4 until the total energy $F$ remains unchanged.

Although, the equation (11), that give us the difference between the old and the new energy value, is large, it is very easily calculated since its most parts are constants for the computation of each Jacobi iteration.

The proofs of the equations (11) and (13) are similarly extracted in a similar way as in [16, 20].

\section{Experimental Results}

In this Section, we show the performance of the proposed method on various synthetic and real images, with different types of contours and shapes. We show the active contour evolving in the original image $\Omega$, and the associated piecewiseconstant approximation of $\Omega$ (given by constants $c_{1}$ and $c_{2}$ ). In our numerical experiments, we generally choose the parameters to be $\lambda_{1}=\lambda_{2}=1$. Only the length parameter $\mu$, which has a scaling role, is not the same in all experiments. If we have to detect all or as many objects as possible and of any size, then $\mu$ should be small. If we have to detect only large objects and not to detect small objects (like points, due to noise), then $\mu$ has to be larger.

First, the segmentation results on a two-phase image (Figure1) are presented. The length term is omitted $(\mu=0)$ since there is no noise. Five different initial conditions were used and all of them converged to the correct solution in a very limited number of iterations. In fact, it is hard to find an initial condition that it does not work. The interior contour was automatically detected without 


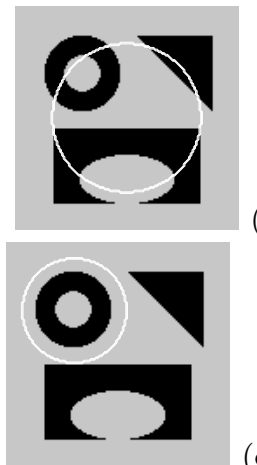

(a)

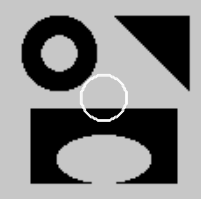

)

(d)

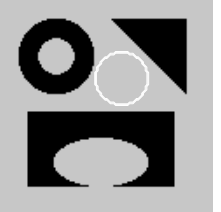

(b)

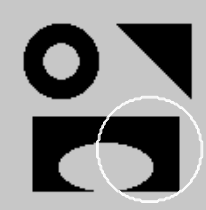

(c)

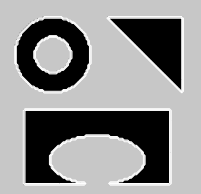

(f)

Fig. 1. Segmentation of a two-phase image. (a), (b), (c), (d) and (e) are five different initial conditions, which have the same result after one sweep. (f) the result segmented image. The interior contour is automatically detected. In this experiment, the length parameter was omitted $(\mu=0)$.
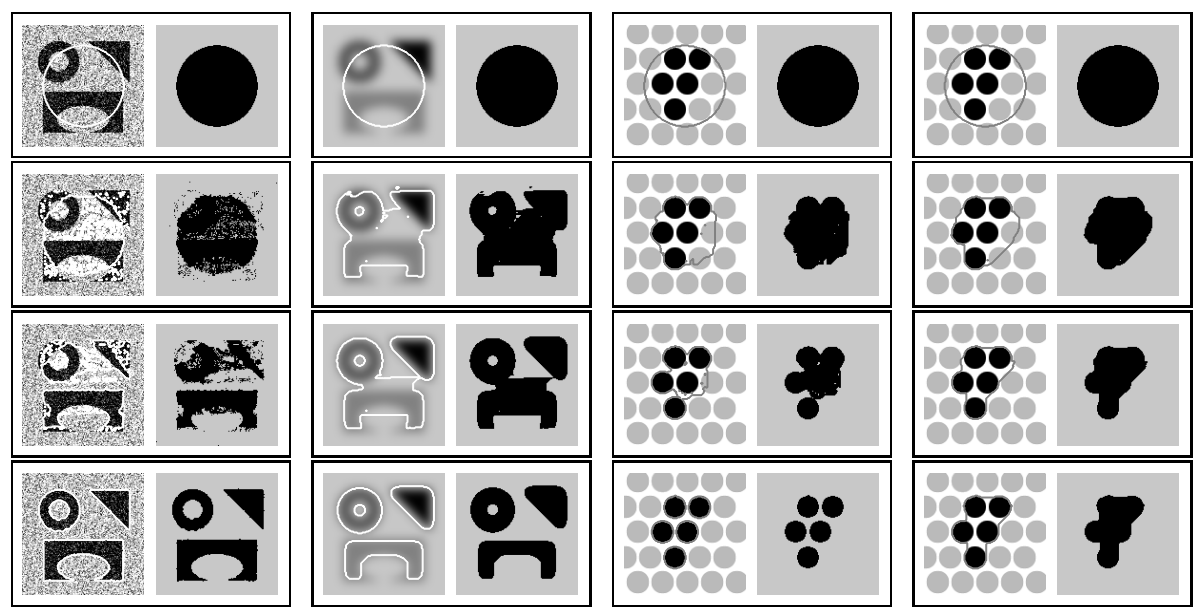

Fig. 2. Detection of different objects from a noisy image with various shapes and with interior contour (first column), blurred objects (second column), geometrically similar objects (third column) and grouping based on chromatic intensity (last column). In all examples, the model converges from the initial (first row) to the final position (last row) with two intermediate steps. The length parameter was set equal to $\mu=3.66$, $\mu=0.61, \mu=0.12$ and $\mu=12.21$ respectively.

considering a second initial model, something that shows the robustness of the algorithm.

The first column of Figure 2 shows how the model works on a noisy synthetic image, with various shapes and an interior contour. The contour is automatically detected, without considering a second initial curve. Jacobi iteration, as well as, the nature of the model allows the automatical change of the topology. Also, the 
length term as well as the terms considering the pixel spatial dependence render the proposed model very robust to noisy images.

The second column of Figure 2 illustrates that the model can detect different objects of different intensities and blurred boundaries. Again, the interior contour of the torus is automatically detected.

In the examples shown in the third and the last column of Figure 2, images with "contours without gradient" or "cognitive contours" (see Chan et al. [15]) are used. The role of the length term as a scale parameter is also illustrated: if $\mu$ is small, then smaller objects will be detected, while as $\mu$ getting larger, then only larger objects are detected, or objects formed by grouping. The last column of Figure 2 depicts how the grouping is based on the chromatic resemblance or identity, among objects of the same shape. Besides, the computation time for the proposed algorithm is much less (real time for images less than $256 \times 256$ ) than other algorithms that solves the Euler-Lagrange equations. Finally, Figure 3 demonstrates how the proposed algorithm could detect object boundaries on real images.

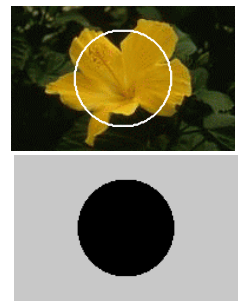

(a)
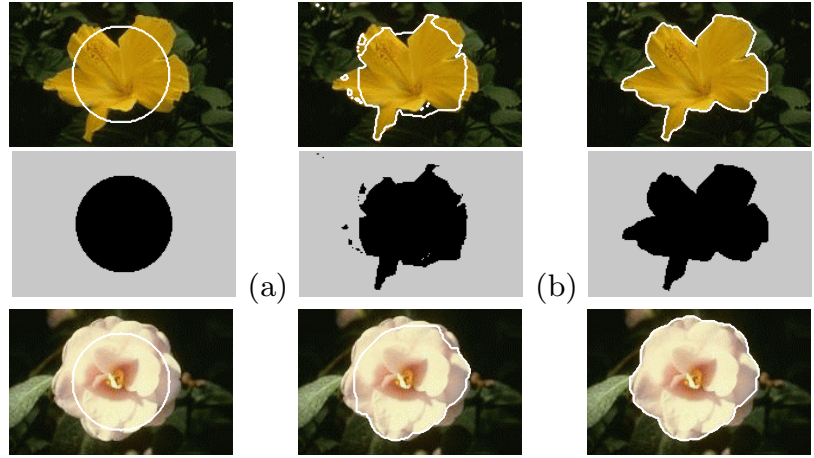

(b)

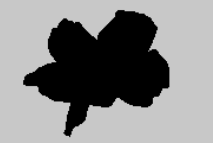

(c)

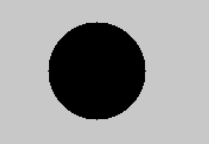

(d)
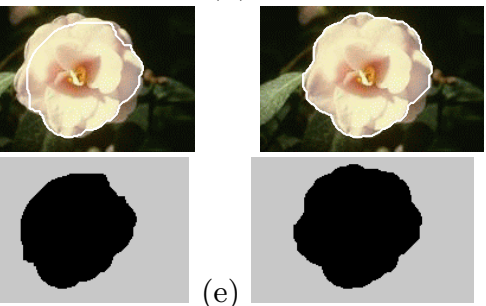

Fig. 3. Detection of object boundaries on real images. The model converges from the initial ((a) and (d)) to the final position ((c) and (f)) with an intermediate step ((b) and $(\mathbf{e}))$. The length parameter was set equal to $\mu=9.77$.

However, the proposed method shares a problem with the other classical methods that solves the Euler-Lagrange equations. There are objects, i.e., texture images, which cannot be detected using only the intensity average only. One way to overcome this difficulty, would be to use other information from the initial image $\Omega$, like the curvature, the orientation of level sets, or any other discriminant. In this framework, we refer the reader to Lopez et al. 21]. 


\section{Conclusion}

In this paper, a novel fast and robust model for active contours to detect objects in an image was introduced. The model can detect objects whose boundaries are not necessarily defined by gradient, due to the fact that it is based on an energy minimization algorithm, and not on an edge-function as the most classical active contour models. This energy is based on fuzzy logic, which can be seen as a particular case of a minimal partition problem, and is used as the model motivation power evolving the active contour until to catch the desired object boundary. Furthermore, the stopping term of the model evolution does not depend on the gradient of the image, as most of the classical active contours, but instead is related to the image color and spatial segments. The fuzziness of the energy provides a balanced technique with a strong ability to reject "weak", as well as, "strong" local minima. Also, it is not needed to smooth the initial images, even if they are very noisy, since the model very well detect and preserve the locations of the boundaries. The interior contours of the objects can be automatically detected, starting only with the initial curve (model). The initial position of the model can be anywhere in the image, and it does not necessarily surrounds the objects to be detected. Finally, the small computation time of the evolution of the model renders the proposed method as a very promising tool even for real time applications. This lies in the fact that, the introduced method does not solve the Euler-Lagrange equation of the underlying problem, but, instead, calculates the fuzzy energy alterations directly without numerical stability constraints. So, it converges to the desired object boundary very fast.

\section{References}

1. Munoz, X., Freixenet, J., Cufi, X., Marti, J.: Strategies for image segmentation combining region and boundary information. Pattern Recognition Letters 24(1), 375-392 (2003)

2. Kass, M., Witkin, A., Terzopoulos, D.: Snakes: Active contour models. International Journal of Computer Vision 1(4), 321-331 (1988)

3. Malladi, R., Sethian, J.A., Vemuri, B.C.: Shape modeling with front propagation: A level set approach. IEEE Transactions on Pattern Analysis and Machine Intelligence 17(1), 158-175 (1995)

4. Osher, S., Sethian, J.A.: Fronts propagating with curvature-dependent speed: Algorithms based on hamilton-jacobi formulation. Journal of Computational Physics 79, 12-49 (1988)

5. Caselles, V., Kimmel, R., Sapiro, G.: Geodesic active contours. International Journal of Computer Vision 22(1), 61-79 (1997)

6. Yezzi, A., Kichenassamy, S., Kumar, A., Olver, P., Tannenbaum, A.: A geometric snake model for segmentation of medical imagery. IEEE Transactions on Medical Imaging 16(2), 199-209 (1997)

7. Tek, H., Kimia, B.: Image segmentation by reaction diffusion bubbles. In: Proceedings of International Conference on Computer Vision, pp. 156-162 (1995)

8. Cohen, L.: Multiple contour finding and perceptual grouping using minimal paths. Journal of Mathematical Imaging and Vision 14(3), 225-236 (2001) 
9. Gunn, S., Nixon, M.: A robust snake implementation: A dual active contour. IEEE Transactions on Pattern Analysis and Machine Intelligence 19(1), 63-68 (1997)

10. Krinidis, S., Chatzis, V.: A physics-based dual deformable model. Journal of Information Hiding and Multimedia Signal Processing 3(1), 100-121 (2012)

11. Dawood, M., Jiang, X., Schäfers, K.P.: Reliable Dual-Band Based Contour Detection: A Double Dynamic Programming Approach. In: Campilho, A.C., Kamel, M.S. (eds.) ICIAR 2004. LNCS, vol. 3212, pp. 544-551. Springer, Heidelberg (2004)

12. Delgado-Gonzalo, R., Thevenaz, P., Seelamantula, C.S., Unser, M.: Snakes with an ellipse-reproducing property. IEEE Transactions on Image Processing 21(3), 1258-1271 (2012)

13. Erdem, C., Tekalp, A., Sankur, B.: Video object tracking with feedback of performance measures. IEEE Transactions on Circuits and Systems for Video Technology 13(4), 310-324 (2003)

14. Xu, N., Bansal, R., Ahuja, N.: Object segmentation using graph cuts based active contours. In: Proceedings of IEEE International Conference on Computer Vision and Pattern Recognition, vol. 2, pp. 46-53 (2003)

15. Chan, T., Vese, L.: Active contours without edges. IEEE Transactions on Image Processing 10(2), 266-277 (2001)

16. Krinidis, S., Chatzis, V.: Fuzzy energy-based active cotnours. IEEE Transactions on Image Processing 18(12), 2747-2755 (2009)

17. Mishra, A.K., Aloimonos, Y., Cheong, L.F., Kassim, A.: Active visual segmentation. IEEE Transactions on Pattern Analysis and Machine Intelligence 34(4), 639-653 (2012)

18. Samson, C., Blanc-Feraud, L., Aubert, G., Zerubia, J.: A level set model for image classification. International Journal of Computer Vision 40(3), 187-197 (2000)

19. Zheng, Q., Dong, E.Q., Cao, Z.L.: Graph cuts based active contour model with selective local or global segmentation. IET Electronis Letters 48(9), 490-491 (2012)

20. Song, B., Chan, T.: A fast algorithm for level set based optimization. Technical Report CAM 02-68, UCLA (2002)

21. Lopez, C., Morel, J.: Axiomatization of shape analysis and application to texture hyper discrimination. In: Proceedings of IEEE Conference on Computer Vision and Pattern Recognition, Berlin, Germany, pp. 646-647 (June 1993) 